\title{
A DESMISTIFICAÇÃO DO USO DOS CONTRASTES RADIOPACOS
}

\author{
DEMYSTIFYING THE USE RADIOPAQUE CONTRAST
}

\author{
Bianca Machado Cruz \\ Universidade Estadual de Ponta Grossa \\ Data de recebimento: $25 / 11 / 2012$ \\ Data da aprovação: 20/05/2012
}

\begin{abstract}
RESUMO
Este trabalho visa expor de modo quantitativo, exploratório-descritivo, prospectivo, e não experimental o uso dos meios de contrastes radiopacos em exames de imagem para fins de diagnósticos. Os meios de contraste são substâncias radio-densas que têm a capacidade de melhorar a visualização e definição das imagens obtidas. O seu uso desperta nos pacientes curiosidade e receio, uma vez que antigamente os contrastes utilizados eram cancerígenos, tóxicos e causavam vários efeitos colaterais. Atualmente as substâncias utilizadas como meios de contraste são mais seguras, entretanto a divulgação dessa informação é restrita e foge da compreensão dos pacientes. Desta forma, por meio desta pesquisa iremos expor dados reais que comprovam que as reações adversas, que antes ocorriam com muita frequência e intensidade, não mais se efetivam, uma vez que, atualmente, são utilizados contrastes de última geração, os quais que só têm a acrescentar na melhora do diagnóstico por imagem, tanto que nossos estudos apontam que a porcentagem de reação alérgica, no ano estudado (setembro de 2010 à setembro de 2011), foi de 0,18\%/ano.
\end{abstract}

Palavras-chave: Contrastes. Reação adversa. Evolução dos contrastes.

\begin{abstract}
This paper presents from a quantitative perspective, an exploratory-descriptive, prospective and non-experimental research about the use of radiopaque contrast media in imaging for diagnostic purposes. Contrast media are radio-dense substances that can improve the visualization and definition of images. Their use in patients arouses curiosity and fear, as contrasts used in the past were carcinogenic, toxic and caused several side effects. Nowadays the substances used as media contrasts are safer but this information is restricted and its comprehension is out of the reach of patients. Therefore, this research will present real data which prove that the adverse reactions that occurred with frequency and intensity do not occur anymore as nowadays modern contrasts which can improve diagnostic imaging are used. An example of this improvement is demonstrated in the percentage of allergic reaction - $0.18 \%$ / year - in the years studied (September 2010 to September 2011).
\end{abstract}

Keywords: Iodine contrast media. Adverse reaction. Evolution of contrasts. 


\section{Introdução}

A prática da radiologia clínica tornou-se possível devido a progressos não só no conhecimento médico, equipamento e diagnóstico, como também na criação dos produtos de contrastes iodados (PCI), que permitiram a visualização dos detalhes das estruturas e órgãos internos, que de outro modo não seriam demonstráveis (SANTOS et.al., 2009).

Os meios de contraste iodados são substâncias radiodensas, capazes de melhorar a definição de imagens obtidas em exames radiológicos, o que se torna necessário em diversos procedimentos como angiografia, tomografia computadorizada e ressonância magnética, entre outros (ARAÚJO, 2007).

A extraordinária tolerância aos PCI modernos foi alcançada através de sucessivos desenvolvimentos farmacológicos. Em 1846, Haschek e Lindenthal procederam a opacificação dos vasos sanguíneos de uma mão amputada, utilizando o contraste de sulfureto de mercúrio e cal, com tempo de exposição à radiação de 57 minutos (SANTOS et al., 2009).

Em 1927, Egas Moniz, prestigiado médico neurologista e neurocirurgião, realizou a primeira angiografia cerebral humana, utilizando como produtos de contraste a suspensão coloidal de dióxido de tório, conhecido popularmente como "torotraste". Este produto de contraste era considerado ideal devido à sua radiopacidade e isotonicidade, fornecendo imagens de boa qualidade e boa tolerância por parte dos pacientes. No entanto, anos mais tarde concluiu-se que o "torotraste" era carcinogênico (SANTOS et al., 2009).

Em 1951 foi sintetizado o ácido benzoico tri-iodado, com base no qual foram sintetizados o acetrizoato e seus derivados, produtos de contraste hiperosmolares, com osmolalidade cinco a oito vezes superior à do plasma (MORCOS; THOMSEN, 2001).

Em 1970, foi sintetizado o primeiro contraste não iônico e o primeiro dímero iônico (GAIVÃO, 2005).

Em 1980, após quase 100 anos de evolução, foram sintetizados os primeiros dímeros não iônicos (THOMSEN; MORCOS, 2000; GAIVÃO, 2005).

Apesar dos significantes avanços dos meios de contraste, à medida que seu uso se generalizou, deparamo-nos, inevitavelmente e com frequência crescente, com as reações adversas no seu uso intravascular (SANTOS et al., 2009).
Segundo definição da Anvisa, reação adversa é "Qualquer resposta a um medicamento que seja prejudicial, não intencional, e que ocorra nas doses normalmente utilizadas em seres humanos para profilaxia, diagnóstico e tratamento de doenças, ou para a modificação de uma função fisiológica" (ANVISA, 2003).

A evolução farmacológica tornou o meio de contraste seguro e por isso amplamente utilizado a fim de garantir a excelência no diagnóstico, preservando e melhorando a qualidade de vida dos pacientes. Considerando que o número de pessoas que utilizam o contraste aumenta, é esperado que o número de reações adversas aumente proporcionalmente, entretanto pudemos constatar, por meio desta pesquisa, que esse número não é significativo.

\section{Materiais e métodos}

O presente estudo, com delineamento quantitativo, exploratório-descritivo, prospectivo, e não experimental, transcorreu em uma renomada clínica de diagnóstico por imagem, situada no Município de Ponta Grossa, Paraná. É uma instituição privada, que realiza um amplo espectro de exames radiológicos como: angiotomografia, densitometria óssea, ecocardiografia e eco de stress, ecocardiograma, transesofágico, mamografia digitalizada, punções e biópsias, radiologia digitalizada, ressonância magnética aberta, ressonância magnética de alto campo, tomografia computadorizada helicoidal, tomografia computadorizada multislice cardíaca. A clínica atende a vários convênios, ao SUS e também a particulares.

Foram escolhidos, entre todos os exames citados acima, somente os de ressonância magnética e tomografia computadorizada, que são justamente os que requerem o uso de contrastes. Os dados foram retirados das planilhas de registros, preenchidas manualmente pela equipe de enfermagem durante todo o horário do funcionamento da clínica. Todos os exames realizados ficam arquivados; assim foi separado para nosso estudo um período de tempo que compreendeu de setembro de 2010 à setembro de 2011. Nesses registros encontramos dados sobre as reações adversas apresentadas pelas pessoas que foram submetidas a exames com utilização de contraste, bem como a classificação de intensidade desses sintomas. Nesse período do estudo, a clínica realizou 12.152 
exames, entre ressonância magnética e tomografia computadorizada, e desse total de pacientes que realizaram os exames apenas 22 apresentaram reações adversas, as quais variavam do grau leve ao moderado, não havendo relatos de reações severas. Foi considerada reação leve quando a sintomatologia apresentada pelo paciente compreendeu náusea, êmese, tosse, calafrios, cefaleia discreta, tontura, alteração do paladar, prurido, tremores, ansiedade, sudorese, leve palidez, congestão nasal, espirros, edema nos olhos e boca, dor no local da injeção do contraste. Já a reação moderada caracteriza-se por sintomas como taquicardia/ bradicardia, hipertensão, reação cutânea pronunciada, hipotensão, dispneia-sibilos, broncoespasmo, laringoespasmo. As reações severas são observadas quando os sintomas oferecem risco de morte, caracterizados por edema de glote, edema agudo e pulmão, choque, arritmia, não responsividade, parada cardiorrespiratória. (OLIVEIRA, et. al., 2009). Com os dados em mãos, processamos as informações através do uso da porcentagem padrão e obtivemos um resultado que apenas comprovou o que já havíamos constatado: o número de pacientes que apresentam reação adversa ao contraste é pouco significativa.

\section{Resultados}

No grupo de 12.152 pessoas que realizaram os exames de tomografia computadorizada e ressonância magnética com contraste radiopaco, no intervalo de 1 ano, foram registradas apenas 22 reações adversas, com sintomas variados, como calafrios, tontura, mal-estar, sudorese, palidez, urticária, prurido, angioedema, tosse, êmese, broncoespasmo e náuseas. $\mathrm{Na}$ maioria das vezes o mesmo paciente relatou mais de um sintoma, como se apresenta no gráfico a seguir:

Todos os pacientes que apresentaram reação se enquadram nas reações leves ou moderadas, não havendo nenhum caso de reação severa. Mesmo esses casos que foram registrados tiveram regressão em um período curto (30 minutos à $1 \mathrm{dia}$ ). Sendo assim, as chances reais de uma pessoas apresentar reação alérgica dos graus leve à moderado são de $0,18 \%$ /ano.
Gráfico 1 - Aponta no eixo de y os sintomas apresentados pelos pacientes que realizaram os exames de ressonância magnética e tomografia computadorizada com uso de contraste. As reações variaram entre leves e moderadas. No eixo de x observa-se a quantidade de vezes que esse sintoma foi relatado pelos pacientes

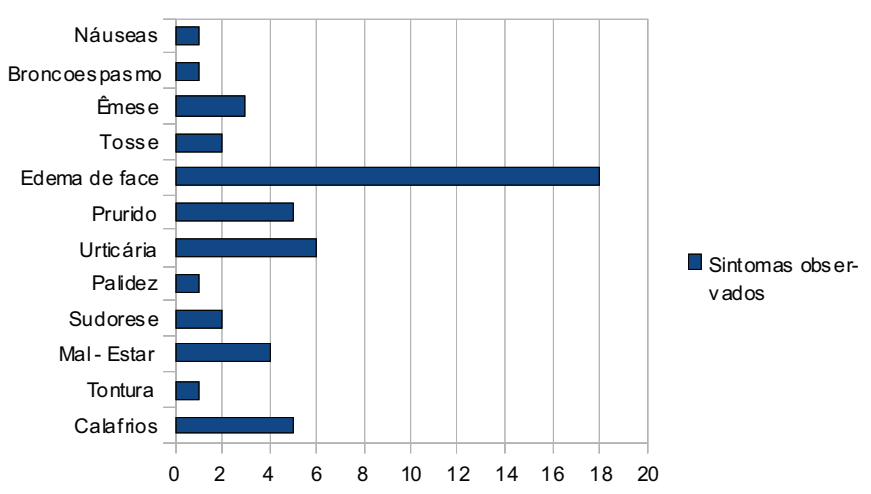

Gráfico 1.1 - Demonstra no eixo y as reações adversas leves, moderadas e severas, e no eixo $\mathrm{x}$ a quantidade de cada tipo de reação. Como se pode observar, o número de reações severas é zero, ou seja, não foi encontrado nenhum registro deste tipo de reação durante todo o período estudado

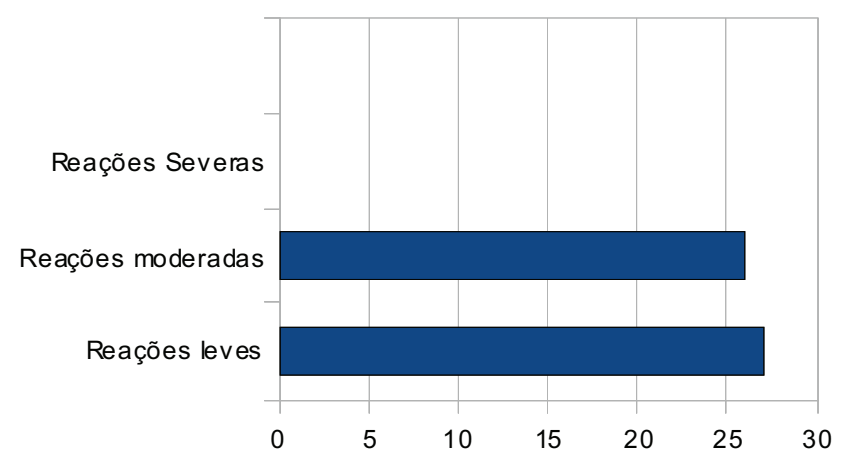

Gráfico 1.2 - Os dados coletados apontaram que, dentre os 22 casos encontrados de reações adversas, 16 deles ocorreram em pacientes do sexo feminino e somente em 6 em pacientes do sexo masculino

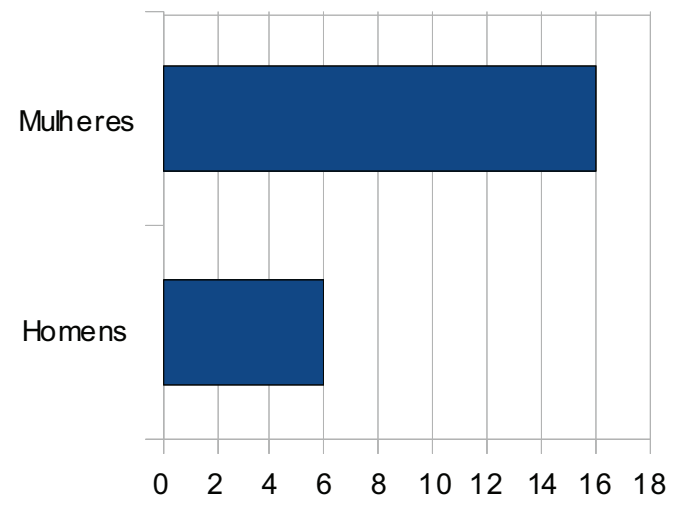




\section{Discussão}

Apesar de, atualmente, a utilização de contrastes serem claramente eficientes na obtenção de imagens com qualidade superior e de graus de rejeição cada vez maiores, o uso do contraste radiopaco causa ainda um certo receio nos pacientes que a ele se submeterão nos seus exames, devido a toda a história da utilização de contrastes já citada anteriormente e que acabou ficando no imaginário das pessoas.

Porém, evidenciam-se cada vez mais as pesquisas e consequente oferta de produtos que funcionam a favor da saúde, ou seja, há uma constante melhoria nas fórmulas utilizadas, com evidentes benefícios para a comunidade.

Percebendo a baixa receptividade dos pacientes em relação ao uso de contraste, seja pela história anterior ou pelo não conhecimento da evolução que ocorreu na área dos fármacos, é que se decidiu demonstrar, por meio do levantamento e processamento de dados do serviço, que o uso desta substância atualmente é seguro à saúde. As chances de acontecer casos de reações adversas nesta clínica é de $0,18 \%$ ao ano. Trazendo esse valor para meses, a chance da reação adversa leve a moderada é de $0,015 \%$ ao mês e indo mais além, se formos pensar nas chances de acontecer uma reação adversa por uso de contraste por dia, considerando um mês que contenha 30 dias, essa reação está na ordem de $0,0005 \%$ ao dia.

Com esses dados expostos, pode-se afirmar que os possíveis usuários do contraste podem ficar menos receosos em relação a sua utilização, levando em consideração que o risco de realmente ocorrer uma reação adversa com este paciente é muito pequena. Também se deve levar em conta o fator da qualidade do exame que é aumentada consideravelmente com a utilização do contraste, proporcionando laudo médico mais seguro, apoiando a decisão clínica, colaborando com a definição diagnóstica e melhora da qualidade de vida do paciente.

Com relação ao último gráfico, onde constatamos que pacientes do gênero feminino apresentaram maior incidência de reações adversas do que o gênero masculino, fazemos duas considerações: a nossa amostra é composta por número maior de mulheres e, segundo dados do Ministério da Saúde, as mulheres têem mais aderência aos serviços de saúde. (GOMES; 2007); (MINISTÉRIO DA SAÚDE; 2008)

\section{Conclusão}

A composição dos meios de contraste radiopaco já produziu efeitos mais acentuados ao organismo humano; entretanto, obteve-se aprimoramento dessas substâncias e assim menos reações foram registradas,como demonstrado por esta pesquisa, em que se observou que as chances de reações adversas no período estudado foi de $0,18 \%$ /ano. Portanto, com esses dados em mãos, a população pode repensar seus conceitos e preconceitos sobre o uso de contrastes radiopacos e levar em consideração as vantagens de sua utilização. Daí a importância de se sanarem as dúvidas e preocupações dos pacientes antes da realização dos seus exames, para que os fatores ansiedade e nervosismo não sejam impasses e elementos de possam comprometer a realização dos serviços.

Levando em consideração que as reações adversas são raras, porém não inexistentes, e que o profissional de saúde mais próximo do paciente no momento da injeção do contraste é o de enfermagem, sugere-se também que esta equipe deva estar apta para o reconhecimento e classificação desses sintomas a fim de assegurar a imediata e eficiente assistência.

\section{Referências}

ARAÚJO, A. Tomografia computadorizada: princípios físicos e aplicações. São Paulo: Corpus, 2007. p.13-14.

ANVISA. Ministério da Saúde. Disponível em: $<$ http://portal.anvisa.gov.br/wps/portal/anvisa/posuso/ farmacovigilancia/!ut/p/c5/04>. Acesso em: 22 set. 2011

GAIVÃO, F. M. Imagiologia clínica: princípios e técnicas. Coimbra: Serviço de Imagiologia dos Hospitais da Universidade de Coimbra, 2005.

GOMES R. et.al. Por que os homens buscam menos os serviços de saúde do que as mulheres? As explicações de homens com baixa escolaridade e homens com ensino superior. Cad. Saúde Pública, Rio de Janeiro, v.23, n.3, p.565-574, mar, 2007.

JUCHEM, B. C.; DALL'AGNOL, C. M. Reações adversas imediatas ao uso do contraste iodado intravenoso em tomografia computadorizada. Rev Latino-americana Enfermagem, v.15, n.1, 2007.

MARINHO, C. R. Manual de práticas e assistência de enfermagem no setor de diagnóstico por imagem. São Paulo: Iátria, 2010. 
MINISTÉRIO DA SAÚDE. Disponível em: <http://portal. saude.gov.br/portal/saude/visualizar_texto.cfm?idtxt= 33061>. Acesso em: 29 out 2011.

MORCOS, S. K.; THOMSEN, H. S. Adverse reactions to iodinated contrast media. European Radiation 11: 126775. 2001.

NÓBREGA, I. A. Técnicas em Ressonância Magnética Nuclear. 1 ed. São Paulo: Atheneu, 2006

OLIVEIRA, L. A. N. et. al. Assistência à vida em radiologia: guia teórico e prático. São Paulo: Colégio Brasileiro de Radiologia, 2009.

PATRÍCIO, A. F. C. A. et al. Radiologia: atuação do profissional de Enfermagem na área de diagnóstico por imagem. Congresso Brasileiro dos Conselhos de Enfermagem, 13, Paraíba, 2010.

SANTOS, A.; GAIVÃO, A. M.; TAVARES, A.; FERREIRA, S. Produtos de contraste iodado. Acta Médica Portuguesa, v.22, p.261-274, 2009.

THOMSEN, H. S.; MORCOS, S. K. Radiographic contrast media. BJU Internacional 86 (Suppl 1) p.1-10. 2000. 